\title{
Compromiso con la responsabilidad social en Portugal: estudio comparativo entre las áreas metropolitanas de Lisboa y Oporto
}

\author{
María Pache Durán*, María Teresa Nevado Gil*, Inna Sousa Paiva**, Luísa Cagica Carvalho *** \\ *Facultad de Empresa, Finanzas y Turismo, Universidad de Extremadura \\ e-mail: mpache@unex.es | ORCID iD: https://orcid.org/0000-0002-6670-5818 \\ e-mail: tnevado@unex.es | ORCID iD: https://orcid.org/0000-0002-4924-0908 \\ **Instituto Universitário de Lisboa (ISCTE-IUL). \\ e-mail: Inna_Paiva@iscte-iul.pt | ORCID iD: http://orcid.org/0000-0002-9980-7503 \\ ***Instituto Politécnico de Setúbal (CICE-IPS) \& CEFAGE. Universidade de Évora. \\ e-mail: Luísa.c.carvalho@esce.ips.pt | ORCID iD: http://orcid.org/0000-0002-9804-7813
}

Recibido: 24-09-20; 2a versión: 21-10-20; Aceptado: 22-10-20. Publicado: 15-10-21

Cómo citar este artículo/Citation: Pache Durán, M.; Nevado Gil, M. T.; Sousa Paiva, I.; Cagica Carvalho, L. (2021). Compromiso con la responsabilidad social en Portugal: estudio comparativo entre las áreas metropolitanas de Lisboa y Oporto. Revista Española de Documentación Científica, 44 (4), e308. https://doi.org/ 10.3989/redc.2021.4.1838

Resumen: La Responsabilidad Social se ha convertido en una pieza clave para las organizaciones. Al mismo tiempo que la sociedad exige cada vez más el acceso a la información, esto se vuelve particularmente importante en el caso de las organizaciones públicas. El uso de las tecnologías de la información y las comunicaciones (TIC) es una forma fácil y eficaz de acceder a la información para una gran parte de la población. Por lo tanto, las organizaciones deben utilizar este canal de comunicación para informar y comunicarse con su público. Este trabajo tiene por objeto establecer una comparación entre las áreas metropolitanas de Lisboa y Oporto del grado de divulgación de la información sobre Responsabilidad Social que realizan las administraciones públicas locales a través de sus portales web. Con ese fin, se realizó un análisis de contenido aplicando indicadores previamente probados en otros territorios. Los resultados sugieren que, en general, las administraciones públicas locales de las dos áreas metropolitanas están elaborando políticas específicas de Responsabilidad Social que muestran el mismo compromiso con la sostenibilidad, aunque el área metropolitana de Lisboa parece estar más comprometida con la información ambiental.

Palabras clave: divulgación de información; gobierno local; responsabilidad social; portales web; análisis de contenido.

\section{Commitment to social responsibility in Portugal: a comparative study between the metro- politan areas of Lisbon and Porto (Portugal)}

Abstract: Social Responsibility has become a key piece for organizations. At the same time that society is increasingly demanding access to information, this becomes particularly important in the case of public organisations. The use of Information and Communication Technologies (ICT) is an easy and effective way for a large part of the population to access information. Organisations should therefore use this channel of communication to inform and communicate with their audience. This work aims to establish a comparison between the metropolitan areas of Lisbon and Porto of the degree of dissemination of information on Social Responsibility made by local public administrations through their webpages. To this end, a content analysis was carried out applying indicators previously tested in other territories. The results suggest that, in general, local public administrations in the two metropolitan areas are developing specific Social Responsibility policies showing the same commitment to sustainability, although the Lisbon metropolitan area seems to be more committed to environmental information.

Keywords: disclosure of information; local government; social responsibility; webpages; content analysis.

Copyright: (c) 2021 CSIC. Este es un artículo de acceso abierto distribuido bajo los términos de la licencia de uso y distribución Creative Commons Reconocimiento 4.0 Internacional (CC BY 4.0). 


\section{INTRODUCCIÓN}

El sector público se considera un gestor de las cuestiones sociales y ambientales. A diferencia del sector empresarial, el sector público tiene la Responsabilidad Social (RS) y la responsabilidad fiduciaria de conservar los recursos naturales y promover el bienestar social y la equidad. El sector público tiene mayores responsabilidades en la promoción de la noción de desarrollo sostenible en comparación con las empresas (Ball, 2004). En su calidad de principales empleadores, proveedores de servicios y consumidores de recursos, las organizaciones del sector público tienen un impacto significativo en el progreso nacional y mundial hacia el desarrollo sostenible y deberían servir como ejemplo de gestión de la sostenibilidad y de divulgación de información (GRI, 2004, 2005; GRI FPA, 2012).

Los avances tecnológicos, junto con la creciente demanda de información por parte de los ciudadanos, que es cada vez más exigente, mejoran la eficiencia y la eficacia de las administraciones y las acercan al ciudadano (Gichoya, 2005). No hay que olvidar que los ciudadanos contribuyen a la sostenibilidad del sector público, ya que son los principales agentes de interés (López y otros, 2011). Debido al impacto crítico que las entidades del sector público tienen en el medio ambiente y la sociedad, es cada vez más vital explorar sus prácticas de divulgación de la RS, así como realizar investigaciones en las que participen profesionales y organismos políticos (Ball, 2004; Ball y Grubnic, 2010).

La literatura sobre el sector público y la RS se ha centrado principalmente en el contenido de las divulgaciones sociales y ambientales, es decir, las prácticas de presentación de informes sobre la sostenibilidad (Guthrie y Gibson, 1995; Guthrie y Farneti, 2008; Williams y otros, 2011; Goswami y Lodhia, 2014; Williams, 2015; Domingues y otros, 2017), responsable de la presentación de informes sobre sostenibilidad (Marcuccio y Steccolini, 2005; Farneti y Guthrie, 2009; Mussari y Monfardini, 2010; Lodhia y otros, 2012; Lodhia y Jacobs, 2013; Biondi y Bracci,2018), la contabilidad de la sostenibilidad (Ball, 2004) y la participación de los interesados (De Villiers y otros, 2014; Greco y otros, 2015).

Por el contrario, el medio utilizado para la información social y ambiental ha recibido una atención considerablemente menor en la bibliografía, aunque investigaciones anteriores indican que los "medios de comunicación más nuevos", como la World Wide Web, tienen el potencial de mejorar la divulgación y comunicación de la información relacionada con las cuestiones sociales y ambientales (Isenmann y Lenz, 2001, 2002; Cho y otros, 2009).
El objetivo de este estudio es un análisis cualitativo y cuantitativo de la información difundida a través de los portales web de las áreas metropolitanas de Oporto y Lisboa, haciendo una comparación entre ambas. Además, el estudio tiene por objeto sensibilizar al público sobre la importancia de contar con portales de transparencia accesibles en los que los ciudadanos puedan obtener información de cualquier parte del mundo. En este sentido, tenemos que mencionar, tanto para España como para Portugal, la existencia del Índice de Transparencia Municipal, elaborado y publicado en estos últimos años por Transparency International España ${ }^{1}$ y Transparency International Portugal2, orientado a medir el nivel de transparencia de las entidades locales para ofrecer una visión sobre la información que divulgan. El objetivo principal de estas organizaciones es infundir valores de transparencia y rendición de cuentas, así como tratar de propiciar un mayor acercamiento de los ayuntamientos hacia los ciudadanos, impulsando un nivel de transparencia que les permita tener acceso a la información pública.

La metodología aplicada es el análisis de contenido. Según Aceituno, Da Conceição Marques y Ariza (2013) "el análisis de contenido es una de las técnicas básicas para el estudio de la información disponible en línea y se basa en la verificación de una serie de epígrafes entre la información divulgada en el sitio tomando valores dicotómicos (1: presencia de la información buscada, 0: ausencia de la información buscada), procediendo posteriormente a su agregación". Posteriormente, se crean índices para determinar el grado de divulgación de la información sobre la RS en las zonas estudiadas.

Este estudio contribuye a la literatura por las siguientes razones. En primer lugar, se han hecho varios intentos de desarrollar un enfoque específico para la presentación de informes sobre sostenibilidad por parte de los gobiernos locales. Estas estructuras tienen por objeto elaborar indicadores a nivel comunitario en el marco de las actividades de los consejos locales mediante procesos de consulta comunitaria (Wiseman y otros, 2006). La GRI también se esforzó y diseñó el Suplemento del Sector para Organismos Públicos (SSPA) para atender las expectativas de divulgación de los diversos interesados del sector público (GRI, 2005). Los estudios centrados en la aplicación de las directrices de la GRI en el gobierno local han revelado que estas directrices para la presentación de informes tienen un uso limitado debido a la falta de personal capacitado, la falta de recursos y, lo que es más importante, la falta de conocimientos sobre las directrices de la GRI (Sciulli, 2011). Este estudio aporta conocimientos para que los órganos 
reguladores armonicen los indicadores específicos de los gobiernos locales, promoviendo la comparabilidad y la transparencia de la información divulgada. En segundo lugar, en el ámbito académico, el estudio contribuye a una mejor explicación de la divulgación de información sobre la RS en el sector público. También tiene como objetivo completar y enriquecer la literatura existente, añadiendo valor debido a su importancia.

\section{REVISIÓN DE LA LITERATURA}

A lo largo de la historia, el concepto de RS se ha ido expandiendo e incorporando nuevos significados. El Libro Verde de la Comisión Europea define la RS como "la integración voluntaria por parte de las empresas de las preocupaciones sociales y medioambientales en sus operaciones comerciales y en sus relaciones con sus interlocutores" (COM, 2001). También afirma que "ser socialmente responsable no sólo significa cumplir plenamente las obligaciones jurídicas, sino también ir más allá del cumplimiento invirtiendo más en el capital humano, el medio ambiente y las relaciones con las partes interesadas" (COM, 2001).

Las organizaciones divulgan sus actividades de sostenibilidad principalmente para: i) evaluar el estado actual del progreso de una organización hacia la sostenibilidad; y ii) comunicar a las partes interesadas los esfuerzos y los progresos realizados en las diferentes dimensiones de la sostenibilidad (Dalal-Clayton y Bass, 2002; GRI, 2011). Otras razones para la divulgación de la RS que se han reportado en la literatura son (Adams y Mcnicholas, 2007; Daub, 2007): i) evaluación del desempeño en materia de sostenibilidad (SP); ii) comparación con otras organizaciones; iii) facilitar la transparencia y la auditoría externa; iv) convertirse en un líder en la sociedad; v) esfuerzos de comercialización de la sostenibilidad; y vi) promover cambios en la organización.

A pesar de sus avances en el último decenio, el sector público sigue estando a la zaga en cuanto a la divulgación de la RS, si se compara, por ejemplo, con el sector empresarial (Dumay y otros, 2010; Guthrie; Farneti, 2008; Lodhia y otros, 2012). Según Flynn (2012), la presentación de informes del sector público abarca en general los aspectos financieros y el cumplimiento de las normas, incluido el uso eficiente de los recursos financieros y el cumplimiento de los requisitos de los interesados internos.

La literatura identifica que hubo varios llamados a la investigación en RS en el sector público (Gray y otros, 2009). El uso de informes anuales para evaluar las divulgaciones sociales y ambientales no es un fenómeno nuevo y ha sido utilizado por varios investigadores para identificar organizaciones "favorables" (aquellas con amplias divulgaciones sociales y ambientales) en contraposición a las "desfavorables" que tienen divulgaciones limitadas (Campbell, 2000; Moneva y Llena, 2000; Wilmshurst y Frost, 2000).

En el estudio de Farneti y Guthrie (2009) se realizaron entrevistas en siete organismos diferentes del sector público de Australia. El estudio se centró en por qué las organizaciones informan sobre temas de sostenibilidad y no sobre lo que informan. Los resultados sugieren que los informes de sostenibilidad se dirigieron principalmente a los interesados internos. Sin embargo, el informe anual era un dispositivo de información clave para los usuarios externos. Mussari y Monfardini (2010) destacaron que las prácticas de presentación de informes sociales, que son un componente de la presentación de informes sobre sostenibilidad en las administraciones públicas locales italianas, estaban surgiendo y eran impulsadas, principalmente, por razones de legitimidad para recuperar la confianza perdida.

Por su parte, el estudio de Greco y otros (2015) puso de relieve las notables diferencias en las motivaciones y prácticas de presentación de informes sobre RS entre los distintos contextos geográficos. En este estudio comparativo se investigó una muestra de LC italiana y australiana. El estudio llegó a la conclusión de que la motivación para la presentación de informes RS parece estar afectada por diversos valores políticos, sociales y culturales que caracterizan los contextos nacionales en los que operan estas organizaciones. Aunque ha habido una serie de publicaciones académicas que tratan de la RS en el sector público, sus cifras han sido bastante bajas en comparación con las que se centran en la divulgación de la RS. Sin embargo, se han realizado pocos estudios sobre el vínculo entre la divulgación de la RS a través de sitios web en las administraciones públicas locales (Nevado y Gallardo, 2019).

En estudios anteriores se han aplicado los enfoques de análisis de contenido utilizados en la búsqueda de informes impresos para las divulgaciones sociales y ambientales en la web. Los estudios se centraron en países concretos (Craven y Otsmani, 1999; Cormier y Magnan, 2003; Frost y otros 2005; Tagesson y otros, 2009; Midin, Joseph y Mohamad, 2016; Pache y Nevado, 2019; 2020), industrias específicas en países determinados ( $\mathrm{Pa}$ tten, 2002; Lodhia, 2005), así como en regiones como Asia y el Pacífico (Williams y Pei, 1999; Chapple y Moon, 2005), Europa y los Estados Unidos (Maignan y Ralston, 2002) y a nivel mundial (Jose y Lee, 2007; Morhardt, 2010). 
Otros estudios, por su parte, se centraron principalmente en el contenido de la información de los sitios web abordaron cuestiones específicas. Así, Campbell y Beck (2004) realizaron un estudio centrado en el uso de la web por parte de las empresas para responder a las denuncias de negligencia ética, mientras que Coupland (2006) examinó la forma en que las empresas utilizan sus sitios web para elaborar sus propias cuentas de sus responsabilidades sociales y ambientales, legitimando así sus actividades para los interesados. Por su parte, Da Costa Tavares y Rodrigues (2019) analizaron el nivel de divulgación de la RS de las empresas en los informes de sostenibilidad de las entidades del sector público portugués, preparados de conformidad con las directrices de la Iniciativa Mundial de Presentación de Informes (GRI). Estos estudios previamente identificados han hecho contribuciones vitales a la literatura en el ámbito de la comunicación ambiental y social, basada en la web.

En lo que repecta a la divulgación de información a través de sitios web por parte de las administraciones públicas, podemos encontrar números estudios del país vecino. Entre ellos destacan trabajos como los de Navarro y otros (2010), que se centran en el estudio de sitios web de 55 gobiernos locales españoles; García y otros (2013), que analizan 102 gobiernos locales españoles; Nevado y otros,(2013), realizando un estudio de los ayuntamientos extremeños de más de 5.000 habitantes y Pache y Nevado (2019) llevando a cabo un estudio de las 50 capitales de provincias españolas, entre otros. Sin embargo, son más escasos los estudios encontrados en el país objeto de estudio, destacando algunos como los de Nevado y Gallardo (2016), donde se analiza una muestra de municipios que conforman el Alentejo, en Portugal Continental. En este artículo, por tanto, se señala que los estudios deben abordar y analizar la divulgación mediante indicadores de comunicación en la web, además del contenido de la información en los mismos.

\section{SELECCIÓN DE LA MUESTRA}

El crecimiento demográfico y funcional ha dado lugar a un crecimiento en las ciudades, en general, y en Lisboa y Oporto, en particular. Debido a la alta presión demográfica y funcional dentro de la ciudad, surgió un movimiento divergente. En este movimiento es característico que las periferias sean objeto de una demanda creciente, tanto para la construcción de viviendas como para el establecimiento de industrias y servicios. De este modo, están surgiendo zonas que constituyen importantes cuencas de empleo y otras zonas en las que, por el contrario, predomina la función residencial.

Debido a esta creciente demanda de la población y de los sectores de actividad, tradicionalmente urbanos, muchos pueblos y ciudades están en expansión. Las consecuencias de esta expansión son las elevaciones de la ciudad. Esto sucedió con Amadora, Almada, Montijo y Odivelas (a las afueras de Lisboa). Ya en la periferia de la ciudad de Oporto, las mismas situaciones ocurrieron con Vila Nova de Gaia, Maia, Matosinhos y Ermesinde. Así se formaron las áreas metropolitanas de Lisboa y Oporto, a fin de reconocer y promover aún más la existencia de una relación de interdependencia e interacción entre las diversas ciudades de cada área.

El Área Metropolitana de Lisboa es un área metropolitana que abarca 18 municipios de la Gran Lisboa y la Península de Setúbal. Es la zona metropolitana más poblada del país (NUTS ${ }^{3}$ III), con 2.821.876 habitantes (2011), y la segunda región más poblada (NUTS II) después de la Región Norte. Hay 18 municipios que componen el Área Metropolitana de Lisboa, agrupados en dos subregiones: la Gran Lisboa y la Península de Setúbal. Oporto, por su parte, es una ciudad con sus referencias inscritas en la historia, afirmándose hoy como una ciudad tangible, embrionaria de la gran región que es hoy el Área Metropolitana de Oporto (AMP). Situada en la costa norte de Portugal, la AMP abarca un área geográfica compuesta actualmente por 17 municipios contiguos, en un área de aproximadamente $2.040 \mathrm{Km}^{2}$ con una población residente de alrededor de 1.700 .000 habitantes.

\section{METODOLOGÍA}

Para lograr los objetivos del estudio, primero se realizó un análisis descriptivo con el que se difunde la información sobre la RS a través de los sitios web de los municipios de las áreas metropolitanas de Oporto y Lisboa en el año 2019. A partir del objetivo planteado, se plantean las siguientes preguntas de investigación:

P1. ¿Divulgan información responsable los municipios de las áreas metropolitanas de Oporto y Lisboa a través de sus sitios web?

P2. ¿Cuál es el grado de la información divulgada?

P3. ¿Cuál es su naturaleza? En otras palabras, ¿cuál es el tipo de información que se divulga? Las respuestas a estas cuestiones nos darán a conocer la implicación y el compromiso de los municipios de las áreas metropolitanas en relación a los distintos aspectos que se encuentran en relación con la RS.

La justificación de las preguntas de investigación que se plantean tienen su base en la importancia 
Tabla I: Indicadores por dimensiones

\begin{tabular}{|l|l|c|}
\hline Eje de análisis & Definición & No de indicadores \\
\hline Eje de análisis 1 & Información General & 23 \\
\hline Eje de análisis 2 & Información Social & 21 \\
\hline Eje de análisis 3 & Contratación de Servicios y Obras Públicas & 17 \\
\hline Eje de análisis 4 & Información Económica & 19 \\
\hline Eje de análisis 5 & Información Ambiental & 19 \\
\hline Total de indicadores & & $\mathbf{9 9}$ \\
\hline
\end{tabular}

Fuente: Adaptado de Nevado y otros (2013)

de la difusión de información a través de los sitios web como mecanismo de transparencia en la administración pública. El énfasis en la aplicación de la transparencia se introdujo en la legislación portuguesa a partir de las Directivas 2004/17/CE y 2004/18/CE de la Unión Europea. Dichas Directivas se refieren a los procedimientos de contratación pública, que se han previsto para el respeto y la intención de algunos principios, entre ellos los de transparencia e integridad (De Oliveira, 2020). En lo que respecta a la divulgación de información en administraciones públicas, en la revisión de la literatura podemos observar diferentes estudios en los que se ven plasmados un crecimiento cada vez mayor del interés de los stakeholders hacia la transparencia (Nevado y otros, 2013; Pache y Nevado, 2019).

Para ello se utilizó la técnica de análisis de contenido empleada en numerosos estudios de esta naturaleza (Andrikopoulos y Kriklani, 2013; Nevado, Gallardo y Sánchez., 2013; Da Bairral, Silva y Alves, 2015; Montecalvo, Farneti y De Villiers, 2018; Pache y Nevado, 2019). El instrumento utilizado para recopilar información fue un cuestionario propuesto por Nevado y otros (2013), pero adaptado para Portugal. El resultado final permitió reunir 99 indicadores (Anexo I), divididos en cinco dimensiones (Tabla I). Además, se realiza un análisis de consistencia interna a través del coeficiente Alfa de Cronbach (Cronbach, 1951) obteniendo un valor de 0,923. Este dato nos indica una fiabilidad alta respecto a los indicadores propuestos (George y Mallery, 2003).

El sistema de puntuación utilizado fue asignar a cada indicador el valor de 1 si la entidad revela la información o 0 si no. Este sistema de puntuación se ha utilizado en estudios empíricos anteriores de naturaleza similar (Gandía y Archidona, 2008; Frías; Rodríguez y García, 2013; Nevado y Gallardo, 2016). En segundo lugar, se hace una comparación entre las dos áreas metropolitanas. Para ello, con la información recogida, elaboramos: índices de revelación por ítem; índices de revelación por dimensiones e índice de revelación total (Tabla II), siguiendo la metodología utilizada en Carvalho y otros (2018). El uso de índices para medir el nivel de información se ha utilizado en los estudios de Gandía y Archidona (2008), Navarro y otros (2010), Navarro y otros (2015) y Beuren y Angonese (2015), entre otros.

Tabla II: Índices

\begin{tabular}{|l|l|c|}
\hline \multicolumn{1}{|c|}{ Índices } & \multicolumn{1}{|c|}{ Concepto } & Expresión \\
\hline $\begin{array}{l}\text { Índice de divulgación por item } \\
\text { (IDI) }\end{array}$ & $\begin{array}{l}\text { Mide el porcentaje de municipios que } \\
\text { forman cada item }\end{array}$ & $I D I_{i}=\frac{\sum_{i=1}^{N}(A i j)}{N} * 100$ \\
\hline $\begin{array}{l}\text { Índice de divulgación por } \\
\text { dimensión (IDD) }\end{array}$ & Mide la divulgación total de cada dimensión & $I D D_{i}=\left(\frac{\sum_{i=1}^{d}\left(I D I_{i}\right)}{d}\right) * 100$ \\
\hline Índice de divulgación total (IDT) & Mide la divulgación total de la muestra & $I D T=\sum_{i=1}^{D}\left(I D D_{i}\right)$ \\
\hline
\end{tabular}

Fuente: Adaptado de Carvalho y otros (2018)

Nota: $D=$ número de dimensiones; Aij = toma el valor de 1 si la característica que define el indicador (i) está presente en el municipio (j) y 0 en caso contrario; $\mathrm{N}$ = número de municipio; $\mathrm{d}$ = número de elementos en cada dimensión. 


\section{RESULTADOS}

Los resultados obtenidos por cada área metropolitana en relación con todos los índices calculados se reflejan en el Anexo I. A partir de los índices de divulgación por ítem (IDI), que miden el porcentaje de municipios que informan sobre cada indicador propuesto, el análisis del Eje 1, información general, puede verse en la Figura 1, que en el área metropolitana de Lisboa el $100 \%$ de los municipios identifican un área dedicada a la RS, mientras que en el área metropolitana de Oporto sólo ocurre en el $47,06 \%$ de los municipios. Por otra parte, el $82,35 \%$ de los municipios del área metropolitana de Lisboa publican información sobre los diferentes órganos ejecutivos, oficinas y sus funciones, y publican el calendario de reuniones de los órganos municipales, frente al 52,94\% del área de Oporto. De igual modo, en el área metropolitana de Lisboa, la persona o el responsable se identifica en el $88,24 \%$ de los casos, muy por encima del área de Oporto, donde sólo lo hace el $17,65 \%$ de sus municipios. También en Lisboa, un alto porcentaje de municipios, concretamente el 70,59\%, publican la Agenda 21, pero en Oporto este porcentaje se reduce considerablemente $(35,29 \%)$.
En caso contrario, se hace con la indicación del responsable del área de sostenibilidad, la divulgación de las prioridades y estrategias a alcanzar en materia de sostenibilidad, la publicación del registro de intereses o conflictos de intereses del presidente y los concejales, la publicación de los gastos de representación de los órganos de gobierno municipal, la publicación de listas de miembros de la oficina de la presidencia y de concejales, la publicación de informes de las oficinas y departamentos técnicos del Ayuntamiento, la publicación de información sobre los procesos de selección de personal y el código de ética o buena gobernanza del municipio, los porcentajes de divulgación son considerablemente más altos en el área metropolitana de Oporto que en Lisboa. Por otra parte, en ambas áreas metropolitanas, todos los municipios publican información sobre los acontecimientos, logros y fracasos registrados por el municipio en esta área, pero ninguno de ellos identifica los compromisos asumidos por el programa del Ayuntamiento.

Centrándonos en el segundo eje de análisis, la información social, como vemos reflejado en la Figura 2, son los municipios del área metropolitana de Lisboa, que alcanzan las cifras más altas en los

Figura 1: Índice de revelación de cada indicador en el eje de análisis 1 (IDI). Comparación entre Lisboa y Oporto

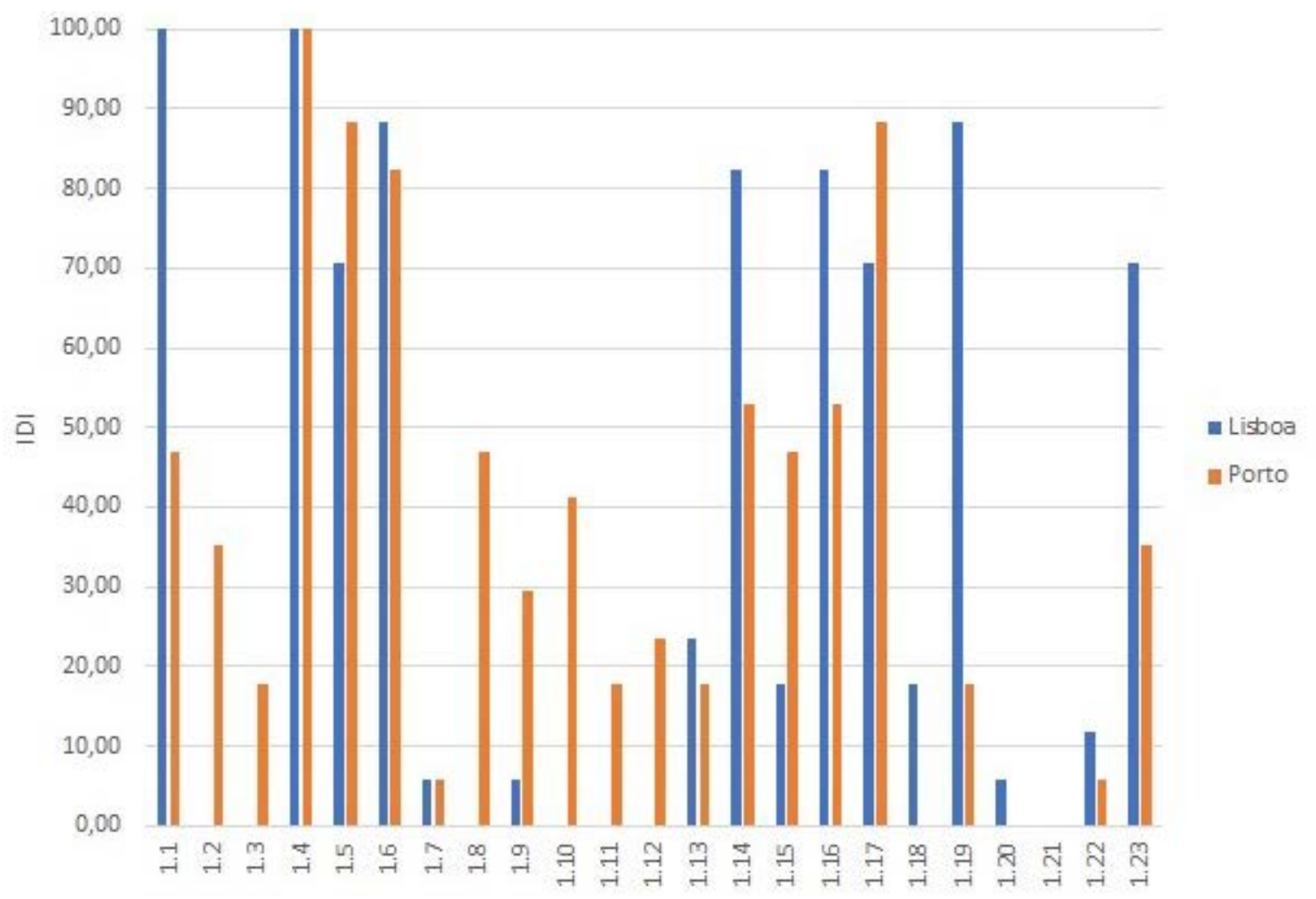


Figura 2: Índice de revelación de cada indicador en el eje de análisis 2 (IDI). Comparación entre Lisboa y Oporto

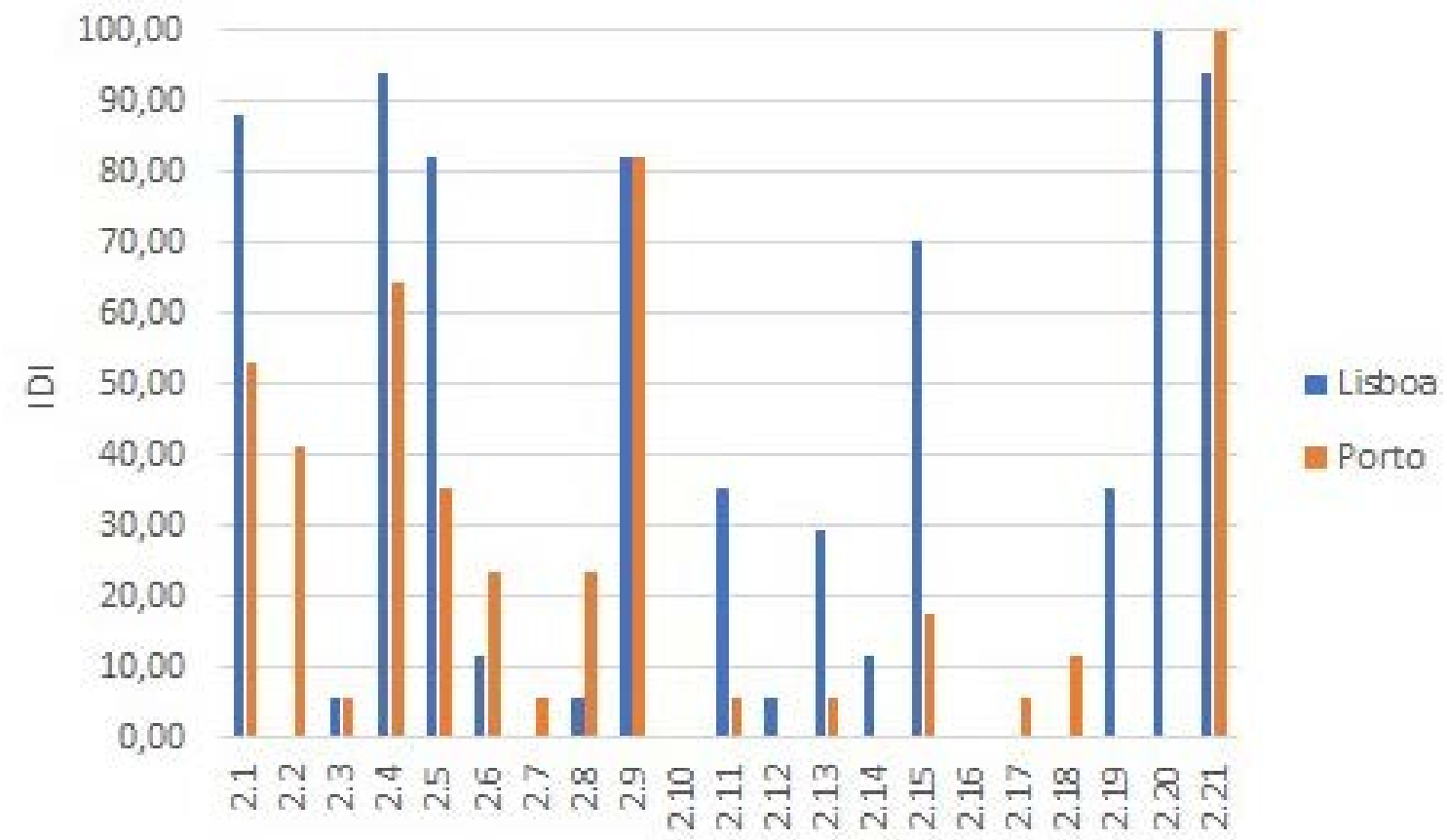

Figura 3: Índice de revelación de cada indicador en el eje de análisis 3 (IDI). Comparación entre Lisboa y Oporto

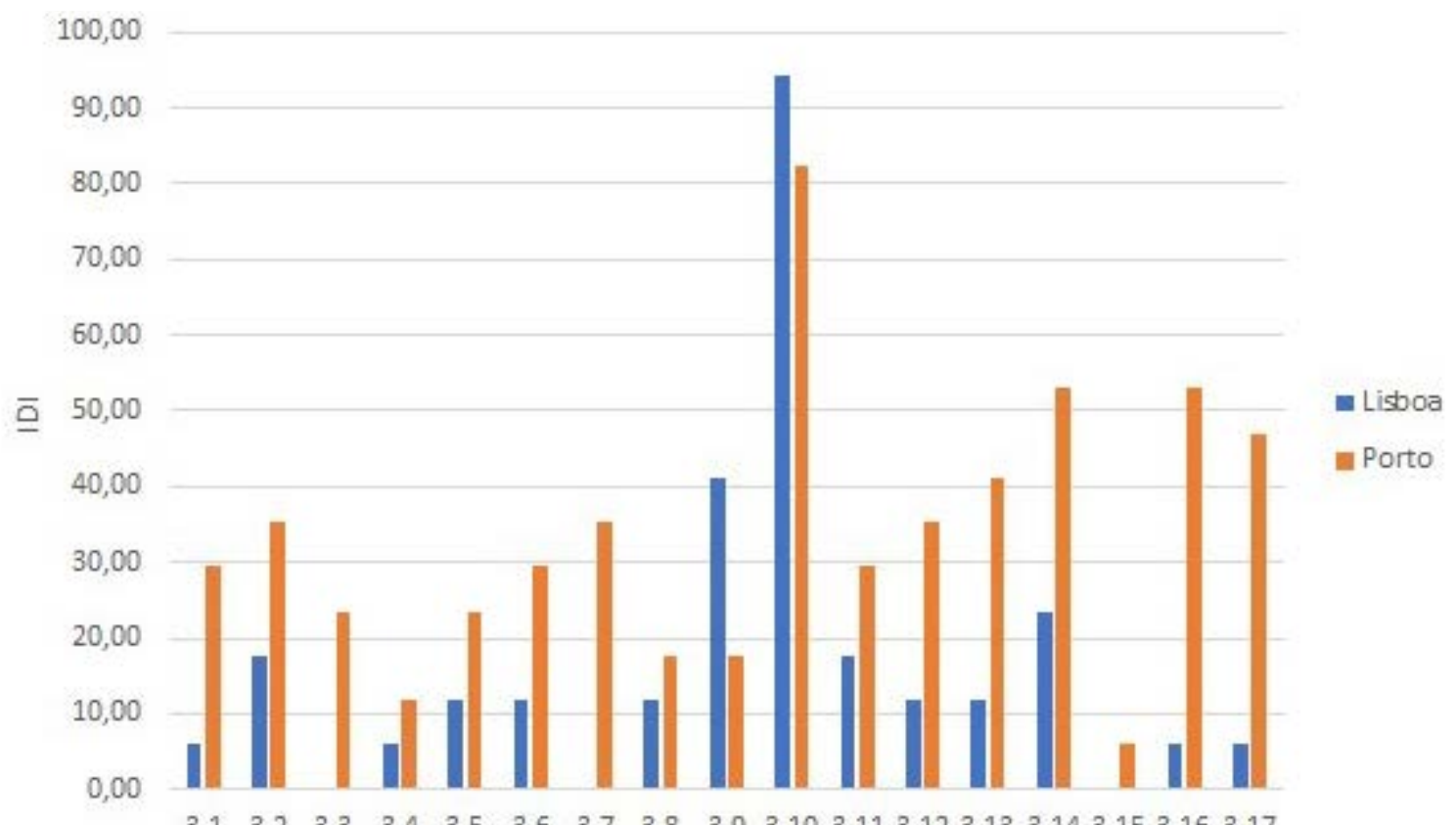


índices relacionados con la existencia de un mapa de portales web de los Ayuntamientos (88,24\% frente al 52,94\% del área de Oporto), enlaces a redes sociales, un sistema de información municipal (concretamente, $88,24 \%, 94,12 \%$ y $82,35 \%$, respectivamente, frente al 52,94\%, 64,71\% y $35 \%$, respectivamente, del área de Oporto). Además, son los que difunden más información sobre aspectos relacionados con el área social $(70,59 \%)$ y todos sus municipios disponen de canales de participación, como foros o servicios de chat.

Por el contrario, los municipios del área metropolitana de Oporto tienen los mayores índices de divulgación en aspectos como la existencia de un motor de búsqueda interna en la página web, la posibilidad de realizar trámites administrativos, autorizaciones o licencias (en línea), la publicación de horarios y precios de equipos/servicios culturales y deportivos, el suministro de información sobre la obtención de premios o distinciones para la RS y la divulgación de información sobre acciones de capacitación en materia de RS para la comunidad.

Para el tercer eje de análisis, la contratación de servicios y obras públicas, la Figura 3 muestra tasas de divulgación muy bajas en ambas regiones metropolitanas, que no superan el $50 \%$ en los in- dicadores propuestos. Se observa que el $94,12 \%$ de los municipios del área metropolitana de Lisboa publican el Plan Director Municipal, mientras que sólo el $82,35 \%$ de los municipios del área metropolitana de Oporto lo hacen. En el resto de los municipios de ambas zonas, la divulgación de información sobre aspectos relacionados con la contratación de servicios es bastante escasa, siendo la zona de Oporto la que más información ofrece.

En cuanto al análisis de la cuarta dimensión, la información económica, se observan valores elevados (Figura 4) en ambas regiones metropolitanas en relación con: la publicación en las portales web de los presupuestos de los municipios, el balance o el balance consolidado, las cuentas de resultados individuales o consolidadas y los informes de gestión $(88,24 \%, 64,71 \%, 64,71 \%$ y $70,59 \%$ respectivamente en las entidades que componen el área metropolitana de Lisboa, frente al 76,47\%, $58,82 \%$ y 47,06 en el área de Oporto).

Las demás tasas son relativamente bajas, a pesar de su importancia. Por ejemplo, sólo el 5,88\% de los municipios del área metropolitana de Lisboa publican listas de préstamos a los bancos y sus respectivos vencimientos. En este caso, el área metropolitana de Oporto la supera con una tasa de

Figura 4: Índice de revelación de cada indicador en el eje de análisis 4 (IDI). Comparación entre Lisboa y Oporto

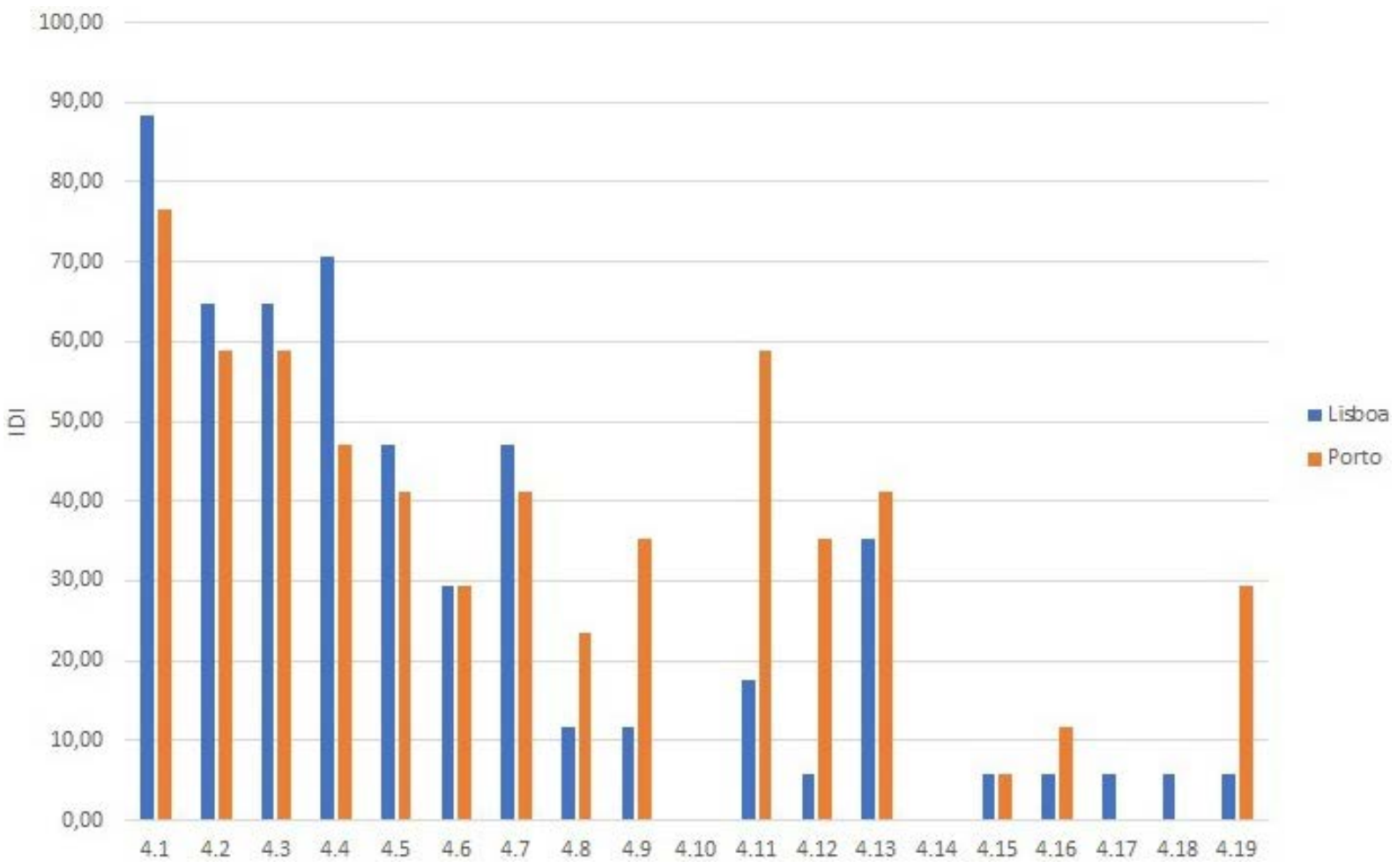


Figura 5: Índice de revelación de cada indicador en el eje de análisis 5 (IDI). Comparación entre Lisboa y Oporto

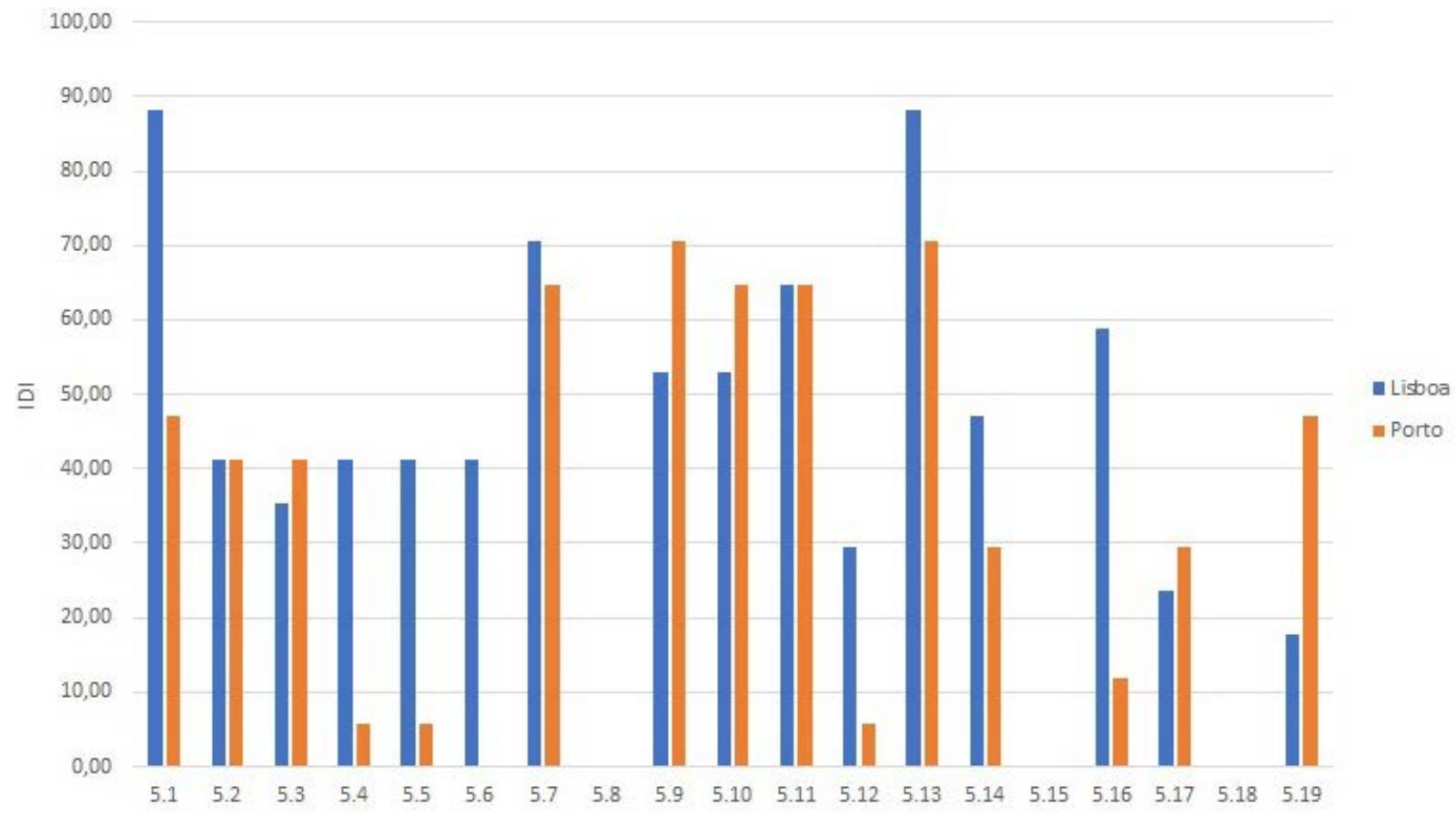

divulgación del 35,29\%. Además, el 5,88\% de los municipios de Lisboa informan sobre la evolución de la deuda, que en este caso coincide con el área metropolitana de Oporto. Ningún municipio de la zona de Oporto publica ingresos fiscales por habitante o gastos per cápita, frente al $5,88 \%$ de los municipios de Lisboa. También hay que mencionar que en ningún municipio se publica información económica importante, como el PIB regional o la tasa de desempleo del municipio.

Finalmente, y siguiendo los índices de divulgación por ítem (IDI), el eje de análisis 5 , la información ambiental, como puede verse en la Figura 5 el compromiso de ambas áreas metropolitanas con la RS en el campo ambiental. El gráfico muestra que los municipios de la zona de Lisboa alcanzan los valores más altos en la mayoría de los indicadores, a saber, en la actualización de la situación ambiental $(88,24 \%)$, la información sobre los vertidos y los destinos de las aguas residuales (70,59\%), las acciones para promover la sensibilidad ambiental de los ciudadanos $(64,71 \%)$ y la información sobre el consumo total de agua $(88,24 \%)$, el resto registra valores inferiores al $50 \%$.

Por otra parte, el área metropolitana de Oporto, aunque con bajos porcentajes, proporciona más información sobre el grado de reducción del impacto ambiental $(41,18 \%)$ e información actualizada so- bre la contaminación del aire y la acústica en las diferentes áreas de cada municipio (47,06\%).

Asimismo, se calcularon los índices de revelación dimensional (IDD), que miden la revelación del total de municipios de las regiones metropolitanas en cada uno de los cinco ejes de análisis propuestos. La Figura 6 muestra cómo el área metropolitana de Lisboa tiene un mayor compromiso con la RS en relación con el área social y ambiental. La situación opuesta se da en las dimensiones de la contratación de servicios y obras públicas y la información económica. Por último, en cuanto a la información general, las dos áreas ofrecen la misma información.

Estos índices de divulgación dimensional (IDD) representan la contribución de cada eje al índice de divulgación total (IDT). La divulgación total de las dos regiones es similar, con tasas de divulgación del $26,87 \%$ en Lisboa y del $26,60 \%$ en Oporto. En el caso del área metropolitana de Lisboa, se observa que la información más importante en materia de IDT es la información ambiental $(7,11 \%)$, en comparación con la información sobre los servicios públicos y la contratación de obras que es la menos difundida $(2,76 \%)$.

El área metropolitana de Oporto ofrece información más general, y su compromiso con las dimensiones de los servicios públicos y la contratación de 
Figura 6: Índice de revelación de tamaño (IDD). Comparación entre Lisboa y Oporto

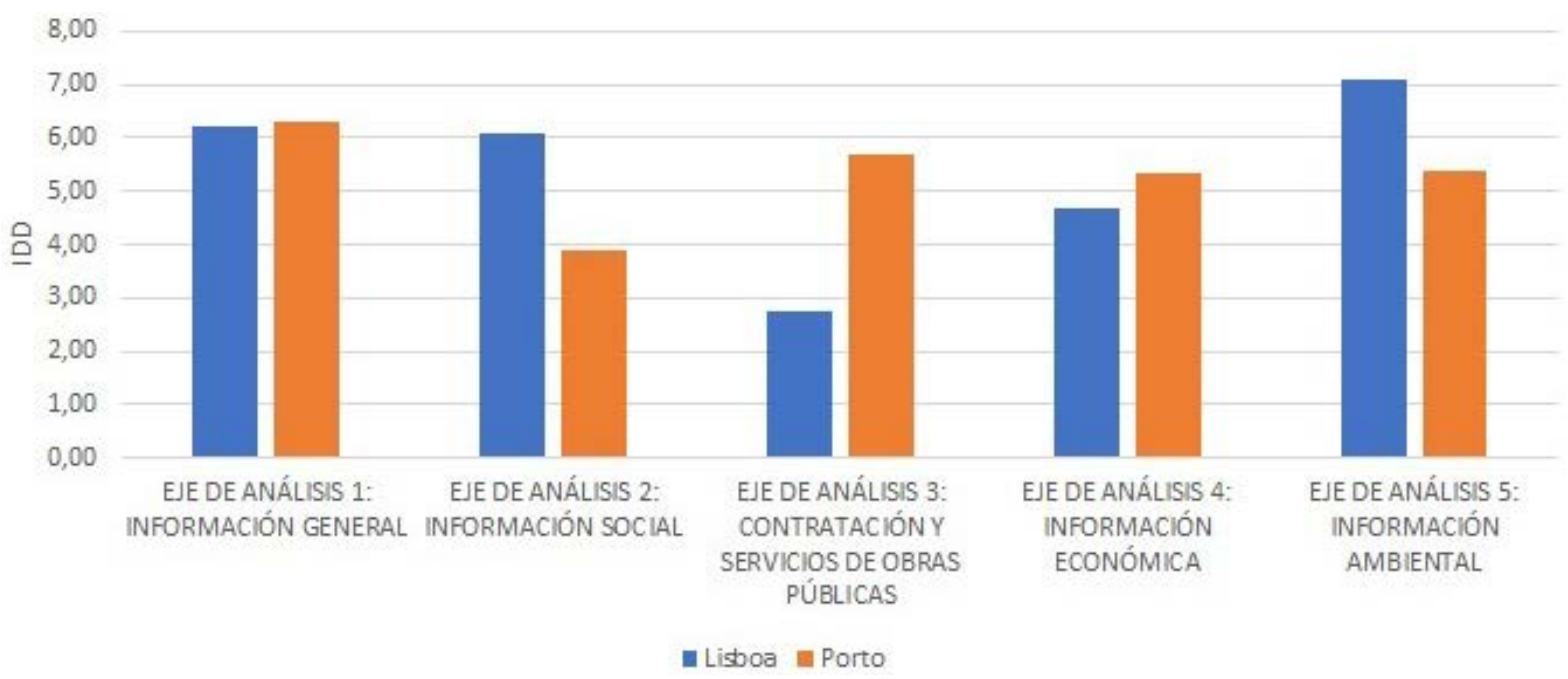

obras, la información económica y ambiental son prácticamente similares. Existen deficiencias en los aspectos sociales, siendo la tasa de divulgación menos difundida $(3,90 \%)$ la que difiere de la del área metropolitana de Lisboa, que ofrece el $6,10 \%$ de la información.

\section{CONCLUSIONES Y FUTURAS LÍNEAS DE INTERÉS}

En este trabajo se realiza un estudio comparativo sobre el compromiso con la RS de las áreas metropolitanas de Lisboa y Oporto, basado en la información divulgada por los residentes de estas regiones a través de sus portales web. Los resultados sugieren que las dos esferas están comprometidas de manera relativamente similar con la elaboración de políticas específicas de RS siendo, tal y como indican los índices de divulgación total calculados (IDT), la tasa de divulgación de Lisboa ligeramente superior $(26,87 \%)$ con respecto a la de Oporto $(26,60 \%)$. A pesar de ello, existe cierta desarticulación en cuanto a la estrategia a largo plazo, por lo que aún queda mucho por hacer en este ámbito. Por lo tanto, en lo que respecta a las preguntas de investigación planteadas de manera inicial, se puede concluir que los municipios de las áreas metropolitanas de Oporto y Lisboa sí divulgan información sobre RS a través de sus portales web, alcanzando un grado de información bajo, si se compara con estudios similares prévios como los de Nevado y Gallardo (2016) (30,69\%), Navarro y otros $(2010)(40,42 \%)$ o Pache y Nevado (2019) $(62,36 \%)$, entre otros.

En cuanto al tipo de información que se divulga, se ha podido ver cómo el área metropolitana de
Lisboa está más comprometida con el medio ambiente. A su vez, destaca el bajo nivel de compromiso social en el área metropolitana de Oporto, en comparación con el área metropolitana de Lisboa. Sin embargo, en los estudios de Nevado y otros (2013), Navarro y otros (2010) y Nevado y Gallardo (2016) se encontró mayor divulgación en los aspectos sociales y una escasa divulgación en lo que respecta a la información medioambiental. Consideramos que uno de los motivos influyentes en la elevada divulgación de información medioambiental de Lisboa a través de su portal web podría ser el hecho de haber sido declarada como Capital Verde Europea otorgado por la Comisión Europea (CE) en 2020, ya que, en los últimos años, la capital ha estado haciendo un gran esfuerzo para mejorar los problemas de sostenibilidad.

El presente estudio revela una serie de informaciones y análisis con implicaciones en el ámbito académico, ya que contribuye a la literatura sobre la divulgación de información sobre la RS en el sector público portugués. Por otra parte, añade valor debido a su importancia práctica y a la identificación de tendencias y áreas menos desarrolladas en este campo. Es evidente la necesidad de realizar estudios regionales que determinen la divulgación de la información en los municipios. Los resultados también serán muy útiles para que los políticos diseñen medidas de apoyo a la divulgación de información social, económica y ambiental.

Somos conscientes de que el estudio no está exento de aspectos que limitan los resultados obtenidos. Por un lado, los efectos de la región y el país deben ser considerados. Las dos áreas metropolitanas están conformadas por sólo 35 munici- 
pios, englobados en un país que alberga a 308 en total, a pesar de que los municipios estudiados se caracterizan por estar densamente poblados. Por ello, la consideración de entidades pertenecientes a otras regiones e incluso a otros países concluiría con resultados más profundos. En consecuencia, y en términos de futuras investigaciones, sería conveniente abordar el estudio con una muestra más amplia que permita realizar análisis comparativos a nivel de países. Por otro lado, podemos señalar que se trata de un estudio meramente descriptivo, de corte transversal, por lo que no es posible realizar inferencias. Por lo tanto, proponemos un estudio explicativo para futuras investigaciones, determinando los factores que podrían influir en estas prácticas de divulgación, así como considerando su evolución a lo largo del tiempo.

\section{NOTAS}

1 Más información disponible en: http://www.transparencia.org.es/. [Consultada a fecha 19/10/2020].

2 Más información disponible en: https://transparencia. pt/itm/. [Consultada a fecha 19/10/2020].

3 Nomenclatura de las Unidades Territoriales Estadísticas (derivado de las siglas en francés de Nomenclature des Unités Territoriales Statistiques).

\section{REFERENCIAS}

Aceituno, J. V. F.; Da Conceição Marques, M.; y Ariza, L. R. (2013). Divulgación de información sostenible: ¿Se adapta a las expectativas de la sociedad? Revista de Contabilidad, 16 (2), 147-158. https://doi.org/10.1016/j.rcsar.2013.07.004

Adams, C. A.; y Mcnicholas, P. (2007). Making a difference: Sustainability reporting, accountability and organisational change. Accounting, Auditing \& Accountability Journal, 20 (3), 382-402. https://doi. org/10.1108/09513570710748553

Andrikopoulos, A.; y Kriklani, N. (2013). Environmental Disclosure and Financial Characteristics of the Firm: The Case of Denmark. Corporate Social Responsibility and Environmental Management, 20 (1), 55-64. https://doi.org/10.1002/csr.1281

Ball, A. (2004). A sustainability accounting project for the UK local government sector?: Testing the social theory mapping process and locating a frame of reference. Critical Perspectives on Accounting, 15 (8), 1009-1035. https://doi.org/10.1016/S1045-2354(02)00209-5

Ball, A.; y Grubnic, S. (2010). Sustainability accounting and accountability in the public sector. In Sustainability Accounting and Accountability. 262-284. Routledge.

Beuren, I. M.; y Angonese, R. (2015). Instruments for determining the disclosure index of accounting information. Revista Eletronica de Estrategia e Negocios-reen, 8 (1), 120-144.

Biondi, L.; y Bracci, E. (2018). Sustainability, popular and integrated reporting in the public sector: A fad and fashion perspective. Sustainability, 10(9), 3112. https://doi.org/10.3390/su10093112
Campbell, D. (2000). Legitimacy Theory or Managerial Reality Construction? Corporate Social Disclosure in Marks and Spencer Plc Corporate Reports 1969-1997. Accounting Forum, 24(1), 80-100.

Campbell, D.; y Beck, A. (2004). Answering allegations: The use of the corporate website for restorative ethical and social disclosure. Business Ethics: A European Review, 13 (2口3), 100-116. https://doi.org/10.1111/ j.1467-8608.2004.00357.x

Carvalho, L. C.; Vázquez, D. G.; y Gil, M. T. N. (2018). Local Municipalities' Involvement in Promoting Entrepreneurship: An Analysis of Web Page Orientation to the Entrepreneurs in Portuguese Municipalities. In: Handbook of Research on Entrepreneurial Ecosystems and Social Dynamics in a Globalized World. 1-19. IGI Global.

Chapple, W.; y Moon, J. (2005). Corporate social responsibility (CSR) in Asia: A seven-country study of CSR web site reporting. Business \& society, 44 (4), 415441. https://doi.org/10.1177/0007650305281658

Cho, C.H.; Phillips, J.R.; Hageman, A.M.; y Patten, D.M. (2009). Media richness, user trust, and perceptions of corporate social responsibility: an experimental investigation of visual website disclosures. Accounting, Auditing and Accountability Journal, 22 (6), 933-952. https://doi.org/10.1108/09513570910980481

Comisión Europea (2001). Libro Verde. Fomentar un Marco Europeo para la Responsabilidad Social de las Empresas, COM/2001/0366, 2001.

Cormier, D.; y Magnan, M. (2003). Environmental Reporting Management: A Continental European perspective. Journal of Accounting and Public Policy, 22 (1), 4362. https://doi.org/10.1016/S0278-4254(02)00085-6

Coupland, C. (2006). Corporate social and environmental responsibility in web-based reports: Currency in the banking sector?. Critical Perspectives on Accounting, 17 (7), 865-881. https://doi.org/10.1016/j. cpa.2005.01.001

Craven, B. M.; y Otsmani, B. (1999). Social and Environmental Reporting on the Internet by Leading UK Companies, Paper presented at the 22nd Annual Congress of the European Accounting Association, Bordeaux, France.

Cronbach, L. J. (1951). Coefficient alpha and the internal structure of tests. Psychometrika, 16 (3), 297-334. doi: https://doi.org/10.1007/BF02310555

Da Bairral, M. A.; Silva, A. H. C.; y Alves, F. J. D. S. (2015). Transparência no setor público: uma análise dos relatórios de gestão anuais de entidades públicas federais no ano de 2010. Revista de Administração Pública, 49 (3), 643-675.

Da Costa Tavares, M. D. C.; y Rodrigues, L. L. (2019). The determinants of sustainability reporting of the Portuguese public sector entities. In Corporate Social Responsibility: Concepts, Methodologies, Tools, and Applications. 653-678. IGI Global.

Dalal-Clayton, B.; y Bass, S. (2002). Sustainable Development Strategies: a Resource Book, Paris. Earthscan Publications Ltd., New York.

Daub, C. H. (2007). Assessing the quality of sustainability reporting: an alternative methodological approach. Journal of Cleaner Production, 15 (1), 75-85. https:// doi.org/10.1016/j.jclepro.2005.08.013 
De Oliveira, B. H. (2020). O Princípio da Transparência na Contratação Pública em Portugal. Análise Crítica do Direito Público Ibero-Americano, 52.

De Villiers, C.; Van Staden, C., Kaur, A.; y Lodhia, S. K. (2014). The state of disclosures on stakeholder engagement in sustainability reporting in Australian local councils. Pacific Accounting Review.

Domingues, A. R.; Lozano, R.; Ceulemans, y K.; Ramos, T. B. (2017). Sustainability reporting in public sector organisations: Exploring the relation between the reporting process and organisational change management for sustainability. Journal of Environmental Management, 192, 292-301. https://doi.org/10.1016/j. jenvman.2017.01.074

Dumay, J.; Guthrie, J.; y Farneti, F. (2010). GRI sustainability reporting guidelines for public and third sector organizations: A critical review. Public Management Review, 12 (4), 531-548. https://doi.org/10.1080/147 19037.2010.496266

Farneti, F.; y Guthrie, J. (2009). Sustainability reporting by Australian public sector organisations: Why they report. Accounting Fórum, 33 (2), 89-98. Taylor \& Francis. https://doi.org/10.1016/j.accfor.2009.04.002

Flynn, N. (2012). Public sector management. Sage Publications.

Frías, J. V.; Rodríguez, L.; y García, I. M. (2013). Is integrated reporting determined by a country's legal system? An exploratory study. Journal of Cleaner Production, 44, 45-55.

Frost, G.; Jones, S.; Loftus, J.; y Van Der Laan, S. (2005). A survey of sustainability reporting practices of Australian reporting entities. Australian Accounting Review, 15 (35), 89-96. https://doi. org/10.1111/j.1835-2561.2005.tb00256.x

Gandía, J. L.; y Archidona, M. C. (2008). Determinants of web site information by Spanish city councils. Online Information Review, 32 (1), 35-57. https://doi. org/10.1108/14684520810865976

García, I. M., Frías, J. V.; y Rodríguez, L. (2013). Determinants of Corporate Social Disclosure in Spanish Local Governments. Journal of Cleaner Production, 39, 6072. https://doi.org/10.1016/j.jclepro.2012.08.037

George, D. ; y Mallery, P. (2003). Using SPSS for Windows step by step: A Simple Guide and Reference (4. ${ }^{a}$ ed.). Boston: Allyn \& Bacon.

Gichoya, D. (2005). Factors affecting the successful implementation of ICT projects in government. The Electronic Journal of e-Government, 3 (4), 175-184.

Global Reporting Initiative (GRI) (2004). Public Agency Sustainability Reporting: A GRI Resource Document In Support of the Public Agency Sector Supplement Project. Global Reporting Intiative.

Global Reporting Initiative (GRI) (2005). Sector Supplement for Public Agencies, Amsterdam: Global Reporting Initiative.

Global Reporting Initiative (GRI) (2011). Sustainability Reporting Guidelines 3.1, Amsterdam, The Netherlands. Retrieved from: https://www.globalreporting.org/resourcelibrary/G3.1-Guidelines-Incl-Technical-Protocol. pdf.
Global Reporting Initiative (GRI) FPA (2012). Integrating sustainability into reporting - An Australian public sector perspective, GRI Focal Point Australia (FPA), Sydney.

Goswami, K.; y Lodhia, S. (2014). Sustainability disclosure patterns of South Australian local councils: a case study. Public Money \& Management, 34 (4), 273-280. https://doi.org/10.1080/09540962.2014.920200

Gray, R.; Dillard, J.; y Spence, C. (2009). Social accounting research as if the world matters: an essay in Postalgia and a new absurdism. Public Management Review, 11 (5), 545-573. https://doi. org/10.1080/14719030902798222

Greco, G.; Sciulli, N.; y D'onza, G. (2015). The influence of stakeholder engagement on sustainability reporting: evidence from Italian local councils. Public Management Review, 17 (4), 465-488. https://doi.org/10.108 $0 / 14719037.2013 .798024$

Guthrie, J.; y Farneti, F. (2008). GRI sustainability reporting by Australian public sector organizations. Public Money \& Management, 28 (6), 361-366.

Guthrie, J.; y Gibson, R. (1995). Recent environmental disclosures in annual reports of the Australian public and private sector organisations. Accounting Forum, 19 (2-3), 111-127.

Isenmann, R.; y Lenz, C. (2001). Customized corporate social and environmental reporting by internet-based push and pull technologies. Eco-Management and $\mathrm{Au}$ diting, 8, 100-110. https://doi.org/10.1002/ema.153

Isenmann, R.; y Lenz, C. (2002). Internet use for corporate environmental reporting: current challengestechnical benefits-practical guidance. Business Strategy and the Environment, 11 (3), 181-202. https:// doi.org/10.1002/bse.329

Jose, A.; y Lee, S. M. (2007). Environmental reporting of global corporations: A content analysis based on website disclosures. Journal of Business Ethics, 72 (4), 307-321. https://doi.org/10.1007/s10551-006-91728

Lodhia, S. (2005). Legitimacy motives for World Wide Web (WWW) environmental reporting: An exploratory study into present practices in the Australian minerals industry. Journal of Accounting and Finance, 4, 1-15.

Lodhia, S.; y Jacobs, K. (2013). The practice turn in environmental reporting. Accounting, Auditing \& Accountability Journal. https://doi. org/10.1108/09513571311327471

Lodhia, S.; Jacobs, K.; y Park, Y. J. (2012). Driving public sector environmental reporting: the disclosure practices of Australian commonwealth departments. Public Management Review, 14 (5), 631-647. https://doi.org /10.1080/14719037.2011.642565

López, M. D. G.; Martínez, A. M. R.; y Oliva, C. V. (2011). Transparencia financiera de los municipios españoles. Utilidad y factores relacionados. Auditoria Pública, 55, 109-116.

Maignan, I.; y Ralston, D. A. (2002). Corporate social responsibility in Europe and the US: Insights from businesses' self-presentations. Journal of International Business Studies, 33(3), 497-514. https://doi. org/10.1057/palgrave.jibs. 8491028 
Marcuccio, M.; y Steccolini, I. (2005). Social and environmental reporting in local authorities: a new Italian fashion?. Public Management Review, 7 (2), 155-176. https://doi.org/10.1080/14719030500090444

Midin, M.; Joseph, C.; y Mohamad, N. (2016). Advancing Sustainable Development in the Public Sector via Stakeholders' Engagement Disclosure Website. Procedia-Social and Behavioral Sciences, 224, 93-100. https://doi.org/10.1016/j.sbspro.2016.05.408

Moneva, J. M.; y Llena, F. (2000). Environmental disclosures in the annual reports of large companies in Spain. European Accounting Review, 9 (1), 7-29. https://doi.org/10.1080/096381800407923

Montecalvo, M.; Farneti, F.; y De Villiers, C. (2018). The potential of integrated reporting to enhance sustainability reporting in the public sector. Public Money \& Management, 38(5), 365-374. https://doi.org/10.108 $0 / 09540962.2018 .1477675$

Morhardt, J. E. (2010). Corporate social responsibility and sustainability reporting on the internet. Business Strategy and The Environment, 19 (7), 436-452. https:// doi.org/10.1002/bse.657

Mussari, R.; y Monfardini, P. (2010). Practices of social reporting in public sector and non-profit organizations: An Italian perspective. Public Management Review, 12 (4), 487-492. https://doi.org/10.1080/14719037.201 0.496262

Navarro, A.; Alcaraz, F. J.; y Ortiz, D. (2010). La divulgación de información sobre responsabilidad corporativa en administraciones públicas: Un estudio empírico en gobiernos locales. Revista de Contabilidad, 13 (2), 285314. https://doi.org/10.1016/S1138-4891(10)70019-4

Navarro, A.; Tirado, P.; Ruiz, M.; y De Los Ríos, A. (2015). Divulgación de información sobre responsabilidad social de los gobiernos locales europeos: El caso de los países nórdicos. Gestión y Política Pública, 24 (1), 229-270.

Nevado, M. T.; y Gallardo, D. (2016). Información sobre Responsabilidad Social contenida en las páginas webs de los ayuntamientos. Estudio en la región del Alentejo. Revista Española de Documentación Científica, 39 (4), 150. https://doi.org/10.3989/redc.2016.4.1353

Nevado, M. T.; y Gallardo, D. (2019). Local government social responsibility: empirical evidence in the region of Extremadura. Investigaciones Regionales, 45(3), 161-179.
Nevado, M.T. Gallardo, D. y Sánchez, M. (2013). La administración local y su implicación en la creación de una cultura socialmente responsable. Prisma Social, 10.

Pache, M.; y Nevado, M. T. (2019). Divulgación de información responsable por los gobiernos locales españoles. Investigación Bibliotecológica, 33(81), 111-134. http:// dx.doi.org/10.22201/iibi.24488321xe.2019.81.58043

Patten, D. M. (2002). The relation between environmental performance and environmental disclosure: a research note. Accounting, Organizations and Society, 27 (8), 763-773. https://doi.org/10.1016/S03613682(02)00028-4

Sciulli, N. (2011). Influences on sustainability reporting within local government. International Review of Business Research Papers, 7 (2), 282-291.

Tagesson, T.; Blank, V.; Broberg, P.; y Collin, S. O. (2009). What explains the extent and content of social and environmental disclosures on corporate websites: a study of social and environmental reporting in Swedish listed corporations. Corporate Social Responsibility and Environmental Management, 16 (6), 352-364. https://doi. org/10.1002/csr.194

Williams, B. (2015). The local government accountants' perspective on sustainability. Sustainability Accounting, Management and Policy Journal. https://doi. org/10.1108/SAMPJ-07-2014-0043

Williams, S. M.; y Pei, C. A. H. W. (1999). Corporate social disclosures by listed companies on their web sites: an international comparison. The International Journal of Accounting, 4 (3), 389-419. https://doi.org/10.1016/ S0020-7063(99)00016-3

Williams, B.; Wilmshurst, T.; y Clift, R. (2011). Sustainability reporting by local government in Australia: Current and future prospects. In Accounting Forum, 35 (3), 176-186. Taylor \& Francis. https://doi.org/10.1016/j.accfor.2011.06.004

Wilmshurst, T. D.; y Frost, G. R. (2000). Corporate environmental reporting: A test of legitimacy theory. Accounting, Auditing \& Accountability Journal, 13 (1), 10-26.

Wiseman, J.; Heine, W.; Langworthy, A.; McLean, N.; Pyke, J., Raysmith, H.; y Salvaris, M. (2006). Developing a Community Indicators Framework for Victoria: The final report of the Victorian Community Indicators Project (VCIP). Melbourne: The Institute of Community Engagement and Policy Alternatives (ICEPA), Victoria University. 


\section{ANEXO I}

\begin{tabular}{|c|c|c|}
\hline EJE DE ANÁLISIS 1: ESTRATEGIA Y ANÁLISIS & IDI LISBOA & IDI PORTO \\
\hline 1. Se identifica un área en la página web dedicada únicamente a la RS & 100,00 & 47,06 \\
\hline 2. Hay una indicación de la persona responsable del área de sostenibilidad & 0,00 & 35,29 \\
\hline $\begin{array}{l}\text { 3. Se dan a conocer las prioridades y estrategias que deben alcanzarse en materia de } \\
\text { sostenibilidad }\end{array}$ & 0,00 & 17,65 \\
\hline $\begin{array}{l}\text { 4. Revela información sobre los acontecimientos, logros y fracasos registrados por el } \\
\text { municipio en este ámbito }\end{array}$ & 100,00 & 100,00 \\
\hline $\begin{array}{l}\text { 5. Se publican los datos biográficos del presidente y de los consejeros (miembros del } \\
\text { ejecutivo) }\end{array}$ & 70,59 & 88,24 \\
\hline 6. Se publican las direcciones de correo electrónico del presidente y de los concejales & 88,24 & 82,35 \\
\hline 7. Los salarios del presidente y de los consejeros se publican & 5,88 & 5,88 \\
\hline $\begin{array}{l}\text { 8. Se publica el registro de intereses o conflictos de intereses del presidente y los } \\
\text { concejales }\end{array}$ & 0,00 & 47,06 \\
\hline 9. Se publican los gastos de representación de los órganos de gobierno municipal & 5,88 & 29,41 \\
\hline 10. Se publican las listas de los miembros de la oficina del Presidente y de los asesores & 0,00 & 41,18 \\
\hline 11. Se publican los informes de las oficinas y departamentos técnicos del ayuntamiento & 0,00 & 17,65 \\
\hline 12. Se publica información sobre los procedimientos de selección de personal & 0,00 & 23,53 \\
\hline 13. Se publican los contratos de prestación de servicio & 23,53 & 17,65 \\
\hline $\begin{array}{l}\text { 14. Se publica información sobre los diferentes órganos del ejecutivo, oficinas y sus } \\
\text { funciones }\end{array}$ & 82,35 & 52,94 \\
\hline 15. Se publica el código de ética o buen gobierno del municipio & 17,65 & 47,06 \\
\hline 16. Se publica el calendario de reuniones de los órganos municipales ( $C M+A M)$ & 82,35 & 52,94 \\
\hline 17. Se publican las actas de las reuniones de los órganos del municipio ( $C M+A M)$ & 70,59 & 88,24 \\
\hline 18. Se publican los acuerdos de los organismos municipales (CM+AM) & 17,65 & 0,00 \\
\hline 19. Identificación de la persona u organismo responsable & 88,24 & 17,65 \\
\hline 20. Se publica el programa de gobernanza & 5,88 & 0,00 \\
\hline 21. Se identifican los compromisos asumidos por el programa gubernamental & 0,00 & 0,00 \\
\hline 22. Se publican los resultados de las elecciones & 11,76 & 5,88 \\
\hline 23. Se publica la Agenda 21 & 70,59 & 35,29 \\
\hline Índice de divulgación de la dimensión (IDD) & 6,22 & 6,30 \\
\hline
\end{tabular}

\begin{tabular}{|c|c|c|}
\hline EJE DE ANÁLISIS 2: INFORMACIÓN SOCIAL & IDI LISBOA & IDI PORTO \\
\hline 1. Existe un mapa de la propia página web del ayuntamiento & 88,24 & 52,94 \\
\hline 2. Existe un buscador interno dentro da página & 0,00 & 41,18 \\
\hline 3. Existe la posibilidad de escuchar la página & 5,88 & 5,88 \\
\hline 4. Existen links para redes sociales & 94,12 & 64,71 \\
\hline 5. Existe un sistema de información del municipio (incidencias en el tráfico, incendios ...) & 82,35 & 35,29 \\
\hline $\begin{array}{l}\text { 6. En la página web existe la posibilidad de realizar trámites administrativos, } \\
\text { autorizaciones o licencias (on line) }\end{array}$ & 11,76 & 23,53 \\
\hline $\begin{array}{l}\text { 7. Existe información sobre los proveedores del município (el correo electrónico / } \\
\text { direcciones de contacto, etc.) }\end{array}$ & 0,00 & 5,88 \\
\hline 8. Se publican los horarios y precios de los equipos culturales y deportivos & 5,88 & 23,53 \\
\hline 9. Existe un buzón para el ciudadano o una sección para quejas y sugerencias & 82,35 & 82,35 \\
\hline
\end{tabular}




\begin{tabular}{|l|c|c|}
\hline \multicolumn{1}{|c|}{ EJE DE ANÁLISIS 2: INFORMACIÓN SOCIAL } & IDI LISBOA & IDI PORTO \\
\hline 10. Se difunde información sobre los cursos de capacitación personal & 0,00 & 0,00 \\
\hline 11. Se publican noticias destacadas & 35,29 & 5,88 \\
\hline 12. Existe un Gabinete de Apoyo al Emprendedor & 5,88 & 0,00 \\
\hline 13. Se publican ofertas de empleo público & 29,41 & 5,88 \\
\hline 14. Hay un presupuesto participativo y una forma de participar a través de la página web & 11,76 & 0,00 \\
\hline 15. Se divulga información sobre aspectos relacionados con el área social & 70,59 & 17,65 \\
\hline 16. Se publican las ayudas y subvenciones concedidas & 0,00 & 0,00 \\
\hline 17. Hay información sobre la obtención de premios o distinciones de la RS & 0,00 & 5,88 \\
\hline 18. Se divulga la información sobre las acciones de formación de la RS para la comunidad & 0,00 & 11,76 \\
\hline 19. Existe un espacio para las asociaciones & 35,29 & 0,00 \\
\hline 20. Existen canales de participación como foros o servicios de chat & 100,00 & 0,00 \\
\hline 21. Se publica un Boletín Municipal & 94,12 & 100,00 \\
\hline Índice de divulgación de la dimensión (IDD) & 6,10 & 3,90 \\
\hline
\end{tabular}

\begin{tabular}{|l|c|c|}
\hline EJE DE ANÁLISIS 3: CONTRATACIÓN DE SERVICIOS Y OBRAS PÚBLICAS & IDI LISBOA & IDI PORTO \\
\hline $\begin{array}{l}\text { 1. Se publican los bienes y servicios adquiridos sin competencia (por ajuste directo } \\
\text { u otros procedimientos), los proveedores y los valores que justifican esta modalidad }\end{array}$ & 5,88 & 29,41 \\
\hline 2. Se publican las licitaciones actuales de bienes y servicios & 17,65 & 35,29 \\
\hline 3. Se publicará la resolución de las propuestas de cada procedimiento de quiebra & 0,00 & 23,53 \\
\hline 4. Se publican los adjudicatarios y las entidades concurrentes & 5,88 & 11,76 \\
\hline 5. Se publican los contratos firmados con adjudicatarios & 11,76 & 23,53 \\
\hline $\begin{array}{l}\text { 6. Se publican informes de seguimiento y/o evaluación del desempeño del proveedor / } \\
\text { proveedor de servicios / contratista. }\end{array}$ & 11,76 & 29,41 \\
\hline 7. Se publican el número de contratos adjudicados por cada proveedor & 0,00 & 35,29 \\
\hline 8. Se publican los informes de auditoria de las entidades de fiscalización & 11,76 & 17,65 \\
\hline 9. Existe una sección con contenidos sobre planificación territorial y urbana & 41,18 & 17,65 \\
\hline 10. Se publica el plan Director Municipal (PDM) (plan de ordenación urbana) & 94,12 & 82,35 \\
\hline $\begin{array}{l}\text { 11. Se publica información georreferenciada (SIG) sobre el uso o destino del suelo y } \\
\text { sus factores condicionantes }\end{array}$ & 17,65 & 29,41 \\
\hline 12. Se publican los planes urbanos y parciales en curso, aprobados y en revisión & 11,76 & 35,29 \\
\hline $\begin{array}{l}\text { 13. Se publican los resultados del debate público de los Planes Municipales de } \\
\text { Ordenación del Territorio }\end{array}$ & 11,76 & 41,18 \\
\hline 14. Se publica el REOT (Informe sobre el estado de la planificación territorial) & 23,53 & 52,94 \\
\hline 15. Se publica información sobre las modificaciones del proyecto & 0,00 & 5,88 \\
\hline 16. Se publican las listas de trueque y venta de terrenos municipales & 5,88 & 52,94 \\
\hline $\begin{array}{l}\text { 17. Se publican las listas de desprendimiento de los bienes municipales de dominio } \\
\text { público }\end{array}$ & 5,88 & 47,06 \\
\hline Índice de divulgación de la dimensión (IDD) & 2,76 & 5,71 \\
\hline
\end{tabular}

\section{EJE DE ANÁLISIS 4: INFORMACIÓN ECONÓMICA}

1. Se divulga el presupuesto del ayuntamiento

2. Se divulga el balance individual o el balance consolidado

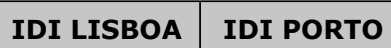

\begin{tabular}{l|l}
\hline 88,24 & 76,47 \\
\hline 64,71 & 58,82 \\
\hline
\end{tabular}




\begin{tabular}{|l|c|c|}
\hline EJE DE ANÁLISIS 4: INFORMACIÓN ECONÓMICA & IDI LISBOA & IDI PORTO \\
\hline 3. Se publican las cuentas de resultados individuales o consolidadas & 64,71 & 58,82 \\
\hline 4. Se publican los informes de gestión & 70,59 & 47,06 \\
\hline 5. Se publican los mapas de flujo de caja & 47,06 & 41,18 \\
\hline 6. Se publican los informes periódicos sobre la ejecución del presupuesto & 29,41 & 29,41 \\
\hline 7. Se publica la ejecución anual del Plan Plurianual de Inversiones & 47,06 & 41,18 \\
\hline 8. Se publican los planes de inversión por parroquia (lista de gastos hecha por parroquia) & 11,76 & 23,53 \\
\hline 9. Se informa sobre las modificaciones presupuestarias & 11,76 & 35,29 \\
\hline $\begin{array}{l}\text { 10. Se publica información económica importante como el PIB o la tasa de desempleo } \\
\text { del municipio }\end{array}$ & 0,00 & 0,00 \\
\hline 11. Se publica la lista de deudas con los proveedores & 17,65 & 58,82 \\
\hline 12. Se publican listas de préstamos a los bancos y sus respectivos vencimientos & 5,88 & 35,29 \\
\hline 13. Se publican las deudas/otros, las deudas a terceros & 35,29 & 41,18 \\
\hline 14. Se publican los índices de endeudamiento por habitante & 0,00 & 0,00 \\
\hline 15. Se proporciona información sobre la evolución de la deuda & 5,88 & 5,88 \\
\hline 16. Se publican las subvenciones recibidas y concedidas & 5,88 & 11,76 \\
\hline 17. Se publican los recibos de impuestos por habitante & 5,88 & 0,00 \\
\hline 18. Se publican los gastos per cápita & 5,88 & 0,00 \\
\hline $\begin{array}{l}\text { 19. Se publican las listas con el valor de los impuestos, tasas, cargos y emolumentos } \\
\text { practicados por el Ayuntamiento }\end{array}$ & 5,88 & 29,41 \\
\hline Índice de divulgación de la dimensión (IDD) & 4,68 & 5,32 \\
\hline
\end{tabular}

\begin{tabular}{|l|c|c|}
\hline EJE DE ANÁLISIS 5: INFORMACIÓN AMBIENTAL & IDI LISBOA & IDI PORTO \\
\hline 1. Información actualizada sobre la situación medioambiental & 88,24 & 47,06 \\
\hline 2. Difusión de iniciativas llevadas a cabo para mitigar impactos ambientales & 41,18 & 41,18 \\
\hline 3. Información sobre el grado de reducción de ese impacto & 35,29 & 41,18 \\
\hline 4. Difusión de acciones llevadas a cabo para incrementar el ahorro de energía & 41,18 & 5,88 \\
\hline 5. Información acerca de iniciativas para promocionar consumo eficiente energía & 41,18 & 5,88 \\
\hline 6. Información sobre el grado de reducción de tales iniciativas & 41,18 & 0,00 \\
\hline 7. Información sobre los vertidos y destinos de aguas residuales & 70,59 & 64,71 \\
\hline 8. Información sobre el total de gastos e inversiones ambientales & 0,00 & 0,00 \\
\hline 9. Información sobre puntos de recogida de basura & 52,94 & 70,59 \\
\hline 10. Información sobre puntos de reciclaje & 52,94 & 64,71 \\
\hline 11. Acciones para promover la sensibilidad de los ciudadanos & 64,71 & 64,71 \\
\hline 12. Información sobre el consumo de energía & 29,41 & 5,88 \\
\hline 13. Información sobre el consumo total de agua & 88,24 & 70,59 \\
\hline 14. Información sobre sanciones e incumplimiento de la legislación medioambiental & 47,06 & 29,41 \\
\hline 15. Información sobre emisiones totales de gases de efecto invernadero & 0,00 & 0,00 \\
\hline 16. Se divulga información sobre políticas ambientales & 58,82 & 11,76 \\
\hline 17. Se divulga información sobre el sistema de gestión medioambiental & 23,53 & 29,41 \\
\hline 18. Existe información sobre la obtención de premios a nivel del medioambiente & 0,00 & 0,00 \\
\hline
\end{tabular}


EJE DE ANÁLISIS 5: INFORMACIÓN AMBIENTAL

19. Existe información actualizada sobre la contaminación del aire y acústica en las distintas zonas del municipio

Índice de divulgación de la dimensión (IDD)
IDI LISBOA

IDI PORTO

17,65

47,06

7,11

5,37 